\title{
THE NONEXISTENCE OF BRANCH POINTS IN THE CLASSICAL SOLUTION OF PLATEAU'S PROBLEM
}

\author{
BY ROBERT OSSERMAN ${ }^{1}$
}

\author{
Communicated by C. M. Morrey, July 17, 1969
}

The result to be proved is the following.

TheORem. Let $C$ be an arbitrary Jordan curve in $R^{3}$. Denote by $\Delta$ the closed unit disk in $R^{2}$. Then there exists a regular minimal surface $S$ of the type of the disk spanning C. Specifically, there exists a map

$$
h: \Delta \rightarrow R^{3}
$$

satisfying

(i) $h$ is continuous in $\Delta$;

(ii) $h$ maps the boundary of $\Delta$ homeomorphically onto $C$;

(iii) each component $h_{k}$ of $h$ is a harmonic function in the interior of $\Delta$, hence the real part of an analytic function $\Phi_{k}$. The functions $\Phi_{k}$ satisfy

$$
\sum_{k=1}^{3}\left(\Phi_{k}^{\prime}\right)^{2} \equiv 0
$$

and

$$
\sum_{k=1}^{3}\left|\Phi_{k}^{\prime}\right|^{2} \neq 0 \text { everywhere. }
$$

(iv) the surface $S$ defined by $h$ has least area among all maps of $\Delta$ into $R^{3}$ satisfying (i) and (ii); if the area of $S$ is infinite, then every interior portion of $S$ has minimum area with respect to its own boundary curve.

It is well known that condition (iii) implies that $h$ is a conformal immersion of the interior of $\Delta$ onto a regular minimal surface. (See for example [1, §II.18].)

The above theorem was proved by Douglas and Radó (see [1, $\$ \S V I .1-7]$ ) except for condition (2). Since the functions $\Phi$ are analytic and not all constant by (ii), it follows that condition (2) can fail at most at isolated points. Such points are called branch points. It has remained an open question whether these branch points actually

AMS Subject Classifications. Primary 49xx, 5304; Secondary 2880.

Key Words and Phrases. Minimal surfaces, Plateau's problem, branch points, area.

1 This research was supported by Army Research Office Contract No. DA31-124 ARO(D)170. 
occur. We show that they do not. Specifically, what we prove is the following.

THEOREM. Any mapping $h$ which satisfies all the conditions of the above theorem except for (2) must also satisfy (2).

The proof depends on a close analysis of the behavior of a minimal surface in the neighborhood of a branch point. The following property is used.

LEMMA. Let $h(w)$ define a minimal surface having a branch point at $w=0$. Then either

(a) there exists $\delta>0$ such that if $0<\left|w_{1}\right|<\delta, 0<\left|w_{2}\right|<\delta$ and $h\left(w_{1}\right)=h\left(w_{2}\right)$, then the tangent planes to $h$ at $w_{1}$ and $w_{2}$ are distinct; or

(b) there exists a local parameter $\tilde{w}$ in a neighborhood of $w=0$ such that $h$ is invariant under some rotation about $\tilde{w}=0$.

Suppose that a solution surface $h$ had a branch point. We may assume that it lies at the origin. Condition (b) of the lemma would contradict property (ii). But using (a) we can replace $h$ in a neighborhood of $w=0$ by a mapping having the same boundary values but strictly smaller area, contradicting (iv).

Details will appear elsewhere.

\section{REFERENCE}

1. T. Rad6, On the problem of Plateau, Vol. 2, Ergebnisse der Mathematik und ihrer Grenzgebiete, Springer-Verlag, Berlin, 1933.

Stanford University, Stanford, California 94305 